\title{
MMP-19 deficiency causes aggravation of colitis due to defects in innate immune cell function
}

\author{
R Brauer $^{1,2,8}$, J Tureckova ${ }^{1,8}$, I Kanchev ${ }^{1}$, M Khoylou $^{3}$, J Skarda ${ }^{4}$, J Prochazka ${ }^{1}$, F Spoutil ${ }^{1}$, IM Beck ${ }^{1}$, \\ O Zbodakova ${ }^{1}$, P Kasparek ${ }^{1,5}$, V Korinek ${ }^{1}$, K Chalupsky ${ }^{1}$, T Karhu ${ }^{6}$, K-H Herzig ${ }^{6}$, M Hajduch ${ }^{3}$, \\ M Gregor ${ }^{1,7}$ and R Sedlacek ${ }^{1}$
}

Matrix metalloproteinases (MMPs) are potential biomarkers for disease activity in inflammatory bowel disease (IBD). However, clinical trials targeting MMPs have not succeeded, likely due to poor understanding of the biological functions of individual MMPs. Here, we explore the role of MMP-19 in IBD pathology. Using a DSS-induced model of colitis, we show

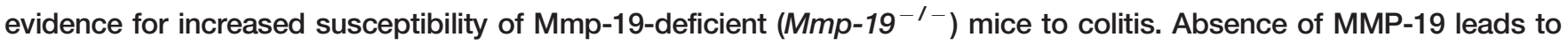
significant disease progression, with reduced survival rates, severe tissue destruction, and elevated levels of proinflammatory modulators in the colon and plasma, and failure to resolve inflammation. There was a striking delay in neutrophil infiltration into the colon of $\mathrm{Mmp}-19^{-1-}$ mice during the acute colitis, leading to persistent inflammation and poor recovery; this was rescued by reconstitution of irradiated $M m p-19^{-1-}$ mice with wild-type bone marrow. Additionally, Mmp-19-deficient macrophages exhibited decreased migration in vivo and in vitro and the mucosal barrier appeared compromised. Finally, chemokine fractalkine (CX3CL1) was identified as a novel substrate of MMP-19, suggesting a link between insufficient processing of CX3CL1 and cell recruitment in the Mmp-19-/- mice. MMP-19 proves to be a critical factor in balanced host response to colonic pathogens, and for orchestrating appropriate innate immune response in colitis.

\section{INTRODUCTION}

Crohn's disease (CD) and ulcerative colitis (UC), the two major types of inflammatory bowel diseases (IBDs), are characterized by continuous or discontinuous mucosal inflammation with inflammatory cell infiltrate, epithelial cell destruction, connective tissue defects, and ulceration of the mucosa. ${ }^{1,2}$ IBD is caused by genetic predisposition, environmental factors, dysregulated immunity, and enteric microbiota, and a combination of these factors results in intestinal injury caused by dysregulated cytokines, nitric oxide, eicosanoids, and proteolytic enzymes.

Matrix metalloproteinases (MMPs) are known to be involved in many physiologic and pathologic processes and they are also upregulated in the context of IBD pathology. ${ }^{3-10}$ The inhibition of MMP activity improves experimental colitis. ${ }^{11,12}$
Unlike most MMPs, MMP-19 is expressed in several healthy tissues, ${ }^{13,14}$ suggesting a role in tissue homeostasis. Epithelial cells of the intestine, ${ }^{15,16}$ peripheral blood mononuclear cells ${ }^{17}$ macrophages, ${ }^{18}$ and keratinocytes ${ }^{19}$ are major producers of this proteinase. MMP-19 processes IGFBP 3 (ref. 19) and various ECM components including laminin $5 \gamma 2$ chain, nidogen-1, tenascin C, and aggrecan. ${ }^{20-23}$ Studies using $M m p$-19-deficient mice revealed its role in the maturation of thymocytes, ${ }^{24}$ accumulation of tenascin- $\mathrm{C}$ in bronchial walls in asthma model, ${ }^{25}$ beneficial effect in liver ${ }^{26}$ and lung ${ }^{27}$ fibrosis, and suppression of tumor angiogenesis and invasion. ${ }^{28,29}$

In this report, we demonstrate that MMP-19 is critically involved in increased susceptibility and exacerbation of colitis, maintaining the epithelial barrier function and regulation of the innate immune response, especially the influx of neutrophils.

${ }^{1}$ Institute of Molecular Genetics of the ASCR, Laboratory of Transgenic Models of Diseases, Prague, Czech Republic. ${ }^{2}$ Women's Guild Lung Institute, Cedars-Sinai Medical Center, Los Angeles, USA. ${ }^{3}$ Institute of Molecular and Translational Medicine, Faculty of Medicine and Dentistry, Palacky University and University Hospital in Olomouc, Olomouc, Czech Republic. ${ }^{4}$ Department of Clinical and Molecular Pathology, Faculty of Medicine and Dentistry, Palacky University and University Hospital in Olomouc, Olomouc, Czech Republic. ${ }^{5}$ Faculty of Sciences, Charles University in Prague, Prague, Czech Republic. ${ }^{6}$ Institute of Biomedicine and Biocenter of Oulu, Medical Research Center Oulu, Oulu University Hospital, Oulu, Finland and ${ }^{7}$ Institute of Molecular Genetics of the ASCR, Laboratory of Integrative Biology, Prague, Czech Republic. Correspondence: M Gregor or R Sedlacek (martin.gregor@img.cas.cz or radislav.sedlacek@img.cas.cz)

${ }^{8}$ These authors contributed equally to this work. 
Neutrophil recruitment is initially delayed; however, Mmp-19-1- mice subsequently develop persistent inflammation. The dysregulated immune response in $\mathrm{Mmp}-19^{-1-}$ mice was rescued by bone marrow transplantation from WT mice. Moreover, we found that MMP-19 directly generates a soluble chemokine domain of CX3CL1 and that Mmp-19 ${ }^{-/-}$mice show diminished processing of CX3CL1. Altogether, MMP-19 mediates beneficial effects in intestinal inflammation and is an important factor in healing and homeostasis of the mucosa.

\section{RESULTS \\ Mmp-19 deficiency results in increased susceptibility to induced colitis}

The expression of MMP-19 significantly increases during the development of colitis (Supplementary Figure 1A online). The experimental model of acute colitis was induced by drinking $2 \%$ DSS (Dextran sulphate sodium) for 6 days. While treatment resulted in significant weight loss in both genotypes, $M m p-19^{-/-}$ mice experienced a greater relative weight loss ( 25 vs. $12 \%$, observed in WT; Figure 1a). This profound difference was associated with a higher disease activity index (DAI) compared to WT mice (4.0 and 2.2 respectively, Figure $\mathbf{1 b}$ ), decreased survival (85\%) compared to WT mice (100\%, Supplementary Figure 1B), and significant reduction in colon length (Figure 1c).

To assess the course of progression of DSS-induced colitis in vivo, signs of inflammation were followed by whole body imaging. Myeloperoxidase (MPO) is an enzyme secreted by neutrophils at the site of inflammation and is widely used as a marker of colitis. To monitor MPO-dependent oxidative stress, luminol-mediated signal was recorded and abdominal areas positive for luminescence were quantified (Figure 1d; for enlarged images see Supplementary Figure 1C). While WT animals showed the peak in inflamed area at days 5 and 6 , in Mmp-19 $19^{-1-}$ mice luminescent signals clearly peaked at day 6 , indicating a delayed influx of MPO-positive neutrophils.

Histological injury scores were quantified from Hematoxylin staining of 'Swiss rolls' of the entire colon (Figure 1e). All DSStreated mice exhibited clear signs of colon inflammation and damage. Extensive lesions in $M m p-19^{-/-}$animals were in striking contrast to fewer lesions in WT mice. Scoring for inflamed lesions, ulceration, and crypt damage in the distal colon revealed more severe injury in $\mathrm{Mmp}-19^{-1-}$ mice (Figure 1f). By day 8, over $80 \%$ of the colon of Mmp-19 $19^{-/}$mice displayed significantly more lesions when compared to all other groups (Figure 1f). Nearly $90 \%$ of the inflamed mucosa displayed ulceration in Mmp-19-1- tissues and, unlike WT animals, the ulcerations affected the entire area of the colonic mucosa (data not shown). Quantification of tissue edema, crypt damage, and ulceration depth (Supplementary Figure 2A) further confirmed exacerbation of colitis in $M m p-19^{-/-}$mice.

\section{Delayed neutrophil recruitment and augmented cytokine expression in $\mathrm{Mmp}^{-19^{-/}}$mice}

Given our findings in DSS-treated $M m p-19^{-/-}$mice (Figure 1), we next quantified neutrophils in histological sections (Figure 2a). Their amount in the inflamed colon tissue of WT mice peaked at day 2 of treatment at approximately $90 \%$ of the total cell count. This was followed by a gradual decrease during the course of the disease, reaching $20 \%$ at day 8 . Conversely, Mmp-19 ${ }^{-/-}$mice exhibited a slow gradual increase in neutrophil numbers that remained elevated compared to WT values at day 8 (Figure 2a; blue (WT) and red (KO) lines). The histological evaluation was supported by a significant increase in myeloperoxidase activity (MPO) at day 8 (Figure $2 \mathbf{b}$ ) correlating with augmented activities of MMP-2 and -9 , two major proteinases produced by neutrophils (Figure 2c). $M m p-19^{-1-}$ mice showed a 5-fold increase of active MMP-9 and a 4-fold increase of active MMP-2 (Figure 2c).

Next we examined whether Mmp-19 deficiency had an impact on inflammatory cells in the peripheral blood and spleen and on inflammatory modulators. DSS-treatment resulted in a 2 -fold increase in $\mathrm{CD} 45+/ \mathrm{CD} 11 \mathrm{~b}+/ \mathrm{Ly} 6 \mathrm{G} / \mathrm{C}+$ granulocytes in the blood of $M m p-19^{-/-}$mice and no differences in the spleen (Figure 2d). To analyze whether development of acute colitis was accompanied by systemic inflammation, cytokine and chemokine levels in the supernatants of colon explant cultures (CEC) and in plasma of DSS-treated mice were determined. Overall, pro-inflammatory Th1 cytokines like TNF- $\alpha$ and IL- 6 were significantly increased in $M m p-19^{-1-}$ mice compared to WT mice (Supplementary Figure 3A,B). Other pro-inflammatory factors such as MCP-1, KC, and G-CSF were also significantly augmented in the Mmp-19-1- CEC cultures (Supplementary Figure 3A), and G-CSF and KC were increased in the plasma of Mmp-19 $19^{-1-}$ mice (Supplementary Figure 3B). Finally, anti-inflammatory Th2 cytokines such as IL- 4 and IL-10 were also significantly increased in the colon of $M m p-19^{-/-}$mice suggesting an ongoing immune suppression activity (Supplementary Figure 3).

\section{Increased epithelial barrier damage in DSS-treated Mmp-19 ${ }^{-/-}$mice}

As the striking deterioration of colitis development in Mmp-19 $19^{-/-}$mice could either mirror dysfunction of immune response or reside in the change of the intestinal barrier function, the barrier permeability in $M m p-19^{-/-}$and WT mice was compared (Figure 3a and $\mathbf{b}$ ). Barrier integrity was assayed by oral gavage of FITC-dextran. Untreated Mmp-19 ${ }^{-/-}$ animals showed higher FITC levels in plasma $(\sim 30 \%$ more than WT mice; Figure 3a) although fluorescence signal was low in WT and Mmp-19-1- mice. Upon DSS administration the permeability for FITC-dextran of intestinal barrier dramatically increased by $\sim 45 \%$ in $M m p-19^{-/-}$mice in comparison to WT animals (Figure $3 \mathbf{b}$ ).

Due to the compromised barrier function in $M m p-19^{-1-}$ mice we examined expression levels of tight junctional constituents. From those assayed (claudins $1,-3,-5,-8$, occludin and ZO-1) only colon specific claudin 8 was significantly decreased in the unchallenged $\mathrm{Mmp}-19^{-/-}$mice (Figure 3c). Immunohistological staining of colon sections confirmed claudin 8 downregulation at the protein level (Figure 3d). In addition, immunohistological analysis of 
a

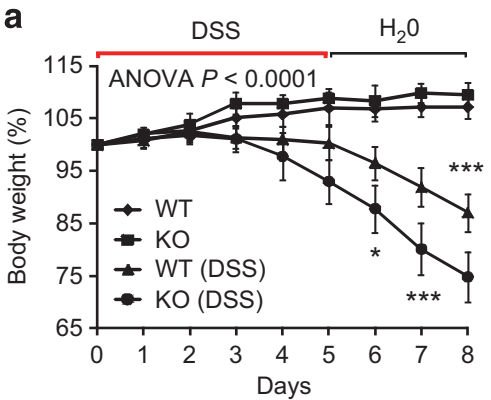

b

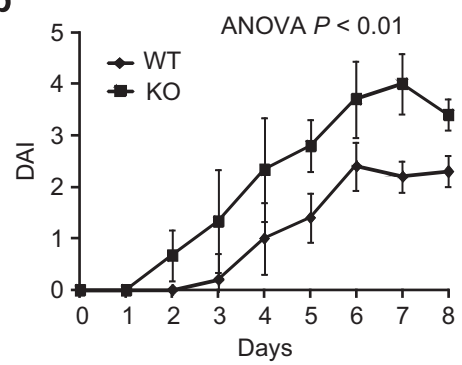

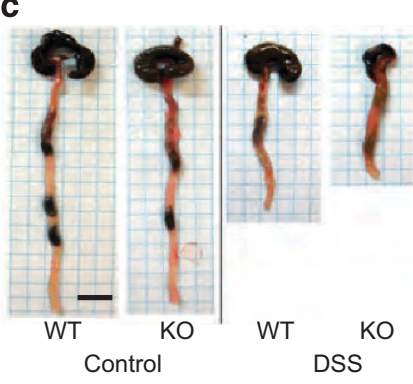

d

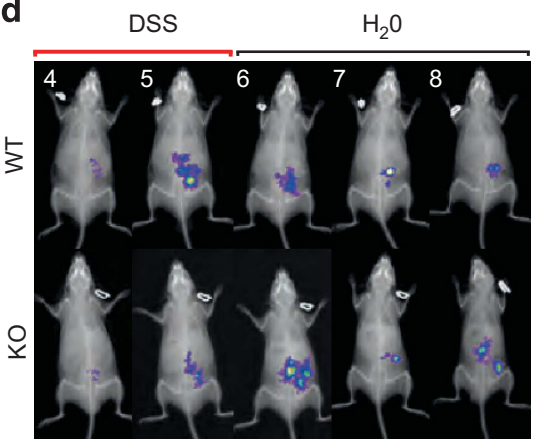

e

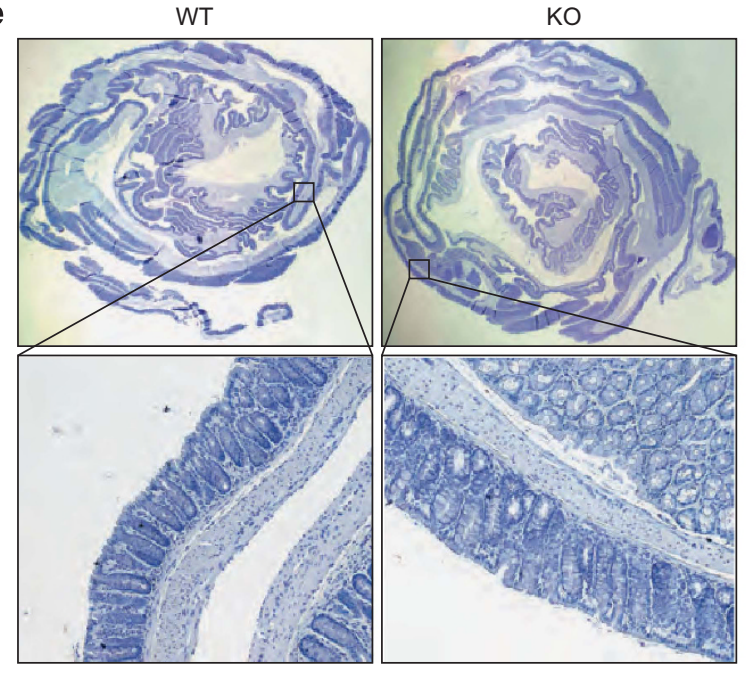

Control
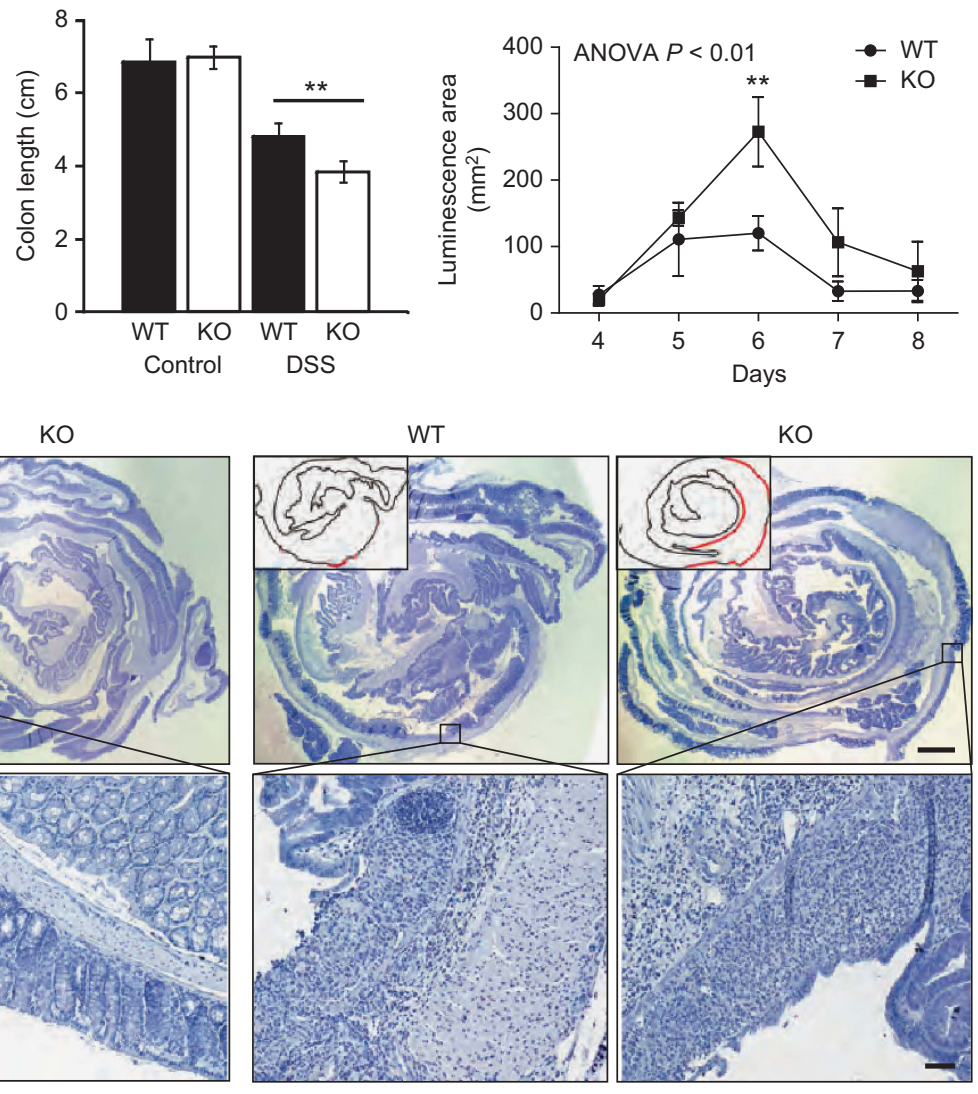

DSS
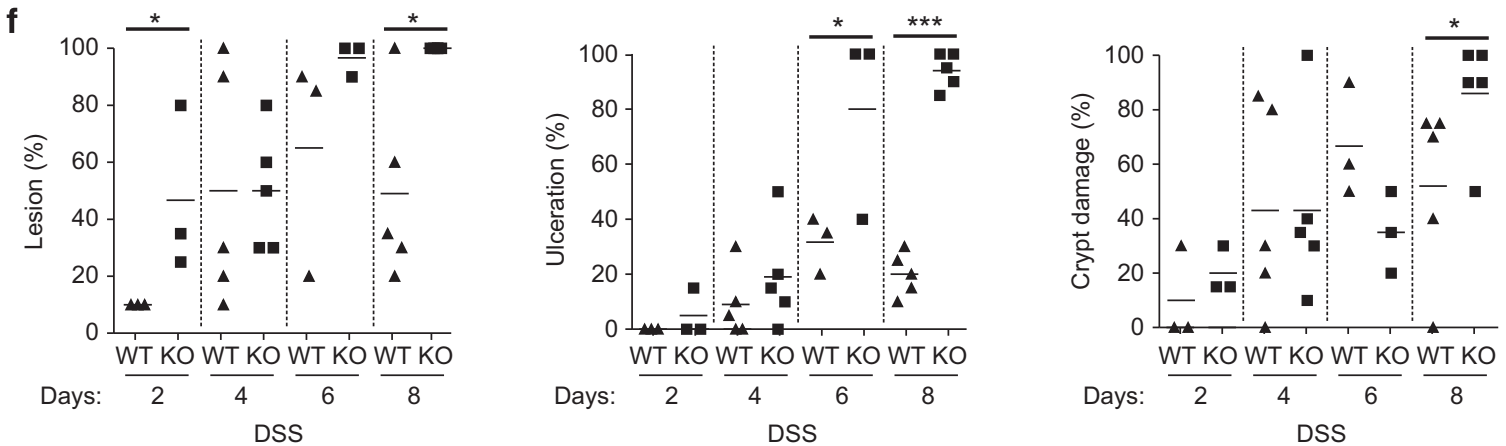

Figure 1 Increased susceptibility of Mmp-19-deficient mice to DSS-induced colitis. (a) Body weight of untreated and DSS-treated mice during the course of acute colitis as a percentage of original weight. $n=9-10$ per genotype. (b) Severity of colitis in DSS-treated mice as disease activity index (DAI). $n=9-10$. (c) Representative images of colon and caecum of healthy (control) vs. DSS-treated (DSS) mice. Scale bar: $1 \mathrm{~cm}$. Colon length of healthy and DSS-treated animals; $n=9-10 ;{ }^{\star \star} P<0.01$, ${ }^{\star \star \star} P<0.001$. (d) Bioluminescent in vivo images of DSS-treated mice. Abdominal areas positive for chemiluminescence signal were quantified. (e) Representative hematoxylin-stained sections of Swiss roll mounts from untreated (control) and DSS-treated mice (day 8). Scale bars: $1 \mathrm{~mm}$ and $50 \mu \mathrm{m}$ (magnified inset). Drawn insets: outlines of lesions (red bars) distributed along the mucosa (black lines) in panels. (f) Quantification of colonic tissue destruction shown as a percentage of tissue area covered with inflammatory lesions, ulceration, and crypt damage, respectively. $n=3 ;{ }^{\star} P<0.05$. 
a
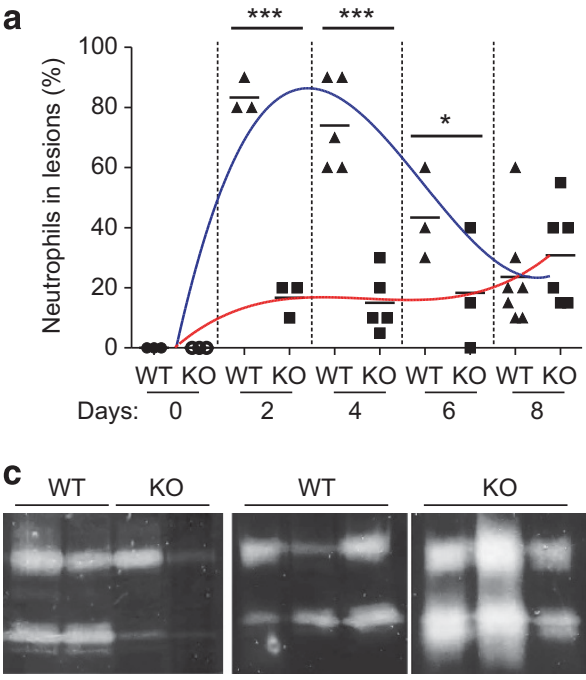

Control
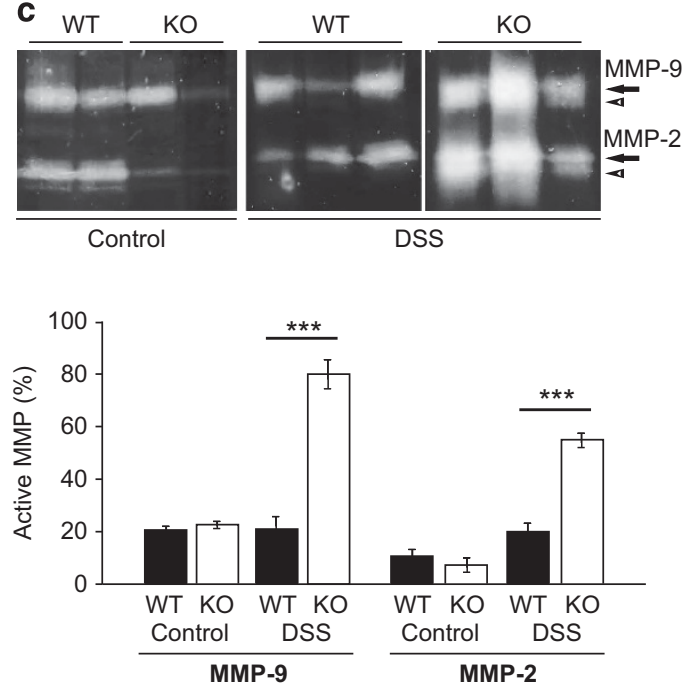

b
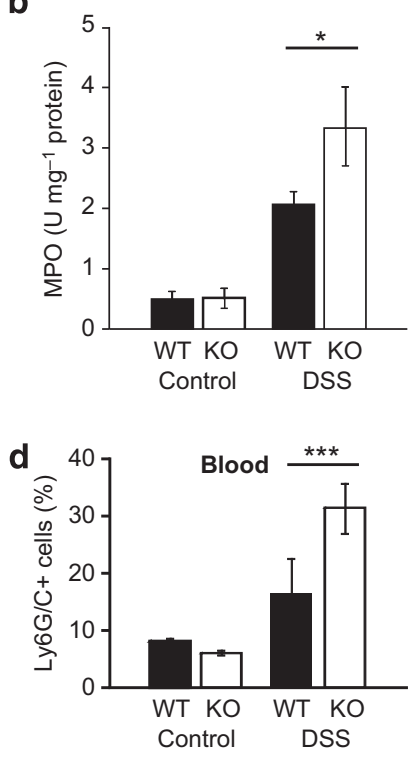

Spleen

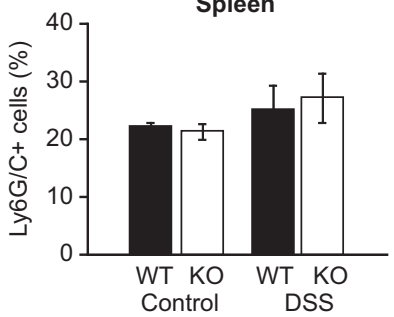

Figure 2 Delayed recruitment of neutrophils at the onset of colitis and their persistence in established disease. (a) Influx of neutrophils in colonic tissue assessed from H\&E stained sections at indicated time points. Fitted plot lines indicate time-course change of neutrophil counts in WT (blue) and Mmp-19-/- (red) mice. $n=3 ;{ }^{\star} P<0.05$, ${ }^{* * *} P<0.001$. (b) MPO activity as marker of neutrophil infiltration in the colon on day $8 ; n=3 ;{ }^{*} P<0.05$. (c) The expression and activity of MMP-2 and MMP-9 analyzed using zymography in colon lysates. Noncontiguous lanes from one DSS experiment are shown. Band intensities were densitometrically determined in two independent experiments. The diagram shows pro-MMP/active MMP ratio. ${ }^{* * \star} P<0.001$. (d) Blood and spleen cells were analyzed by flow cytometry for the granulocyte marker Ly6C/G. $n=4$; ${ }^{* \star \star} P<0.001$. Arrows, pro-MMP; arrowhead, active MMP.

colonic whole mounts revealed reduced secretion of mucin (Figure $3 \mathbf{e}$ and $\mathbf{f}$ ) which indicates a thinner mucus layer. These changes were paralleled by lower counts of PAS-positive goblet cells producing mucin in Mmp-19-1- mice (Figure $3 \mathbf{g}$ and $\mathbf{h}$ ). Together, these data indicate that MMP-19 is involved in regulating intestinal barrier integrity.

\section{Mmp-19 deficiency leads to persistent inflammation}

To study recovery from colitis, mice were treated with $2 \%$ DSS for 5 days followed by a recovery phase (standard drinking water for 10 days). Maximum body weight loss was observed on day 8. WT mice lost less body weight when compared to the Mmp-19-1- mice and re-gained their body weight sooner (Figure 4a). Mmp-19 $19^{-/-}$mice exhibited more severe colitis, impaired recovery (Figure $4 \mathbf{b}$ ) and displayed a significantly shortened colon (Figure 4c). While all WT mice survived, $\sim 40 \%$ of $M m p-19^{-1-}$ animals died during the experiment (Figure 4d).

Histological analysis of the colon revealed highly inflamed and damaged large intestines of $M m p-19^{-/-}$mice, in which approximately $40 \%$ of the mucosa was still affected (Figure 4e,
Supplementary Figure 2B). Mmp-19 $9^{-/-}$mice also exhibited large and deep ulcerations and crypt damage (data not shown, Supplementary Figure 2B), high influx of neutrophils into the colon wall lesions and increased edema (Supplementary Figure 2). These data show that $M m p-19^{-/-}$mice have a reduced capability to recover from colitis.

\section{Immune response in $\mathrm{Mmp}-19^{-/-}$mice develops characteristics of systemic inflammation}

In the chronic phase of colitis, the body weight loss in Mmp-19-1- mice displayed similar characteristics as the acute and recovery phases and $M m p-19^{-/-}$mice never fully recovered (Supplementary Figure 4A,B). The severity of the induced colitis resulted in a $50 \%$ death rate of $\mathrm{Mmp}-19^{-1-}$ mice whereas no deaths were recorded in WT controls (Supplementary Figure 4C). Histological analysis revealed severe damage of the colon wall of $M m p-19^{-/-}$mice (Supplementary Figure 4D), which was paralleled by significantly reduced colon length (Supplementary Figure 4E). Quantification of inflamed lesions, ulceration, and crypt damage demonstrated more profound histological changes in Mmp-19-deficient tissue 

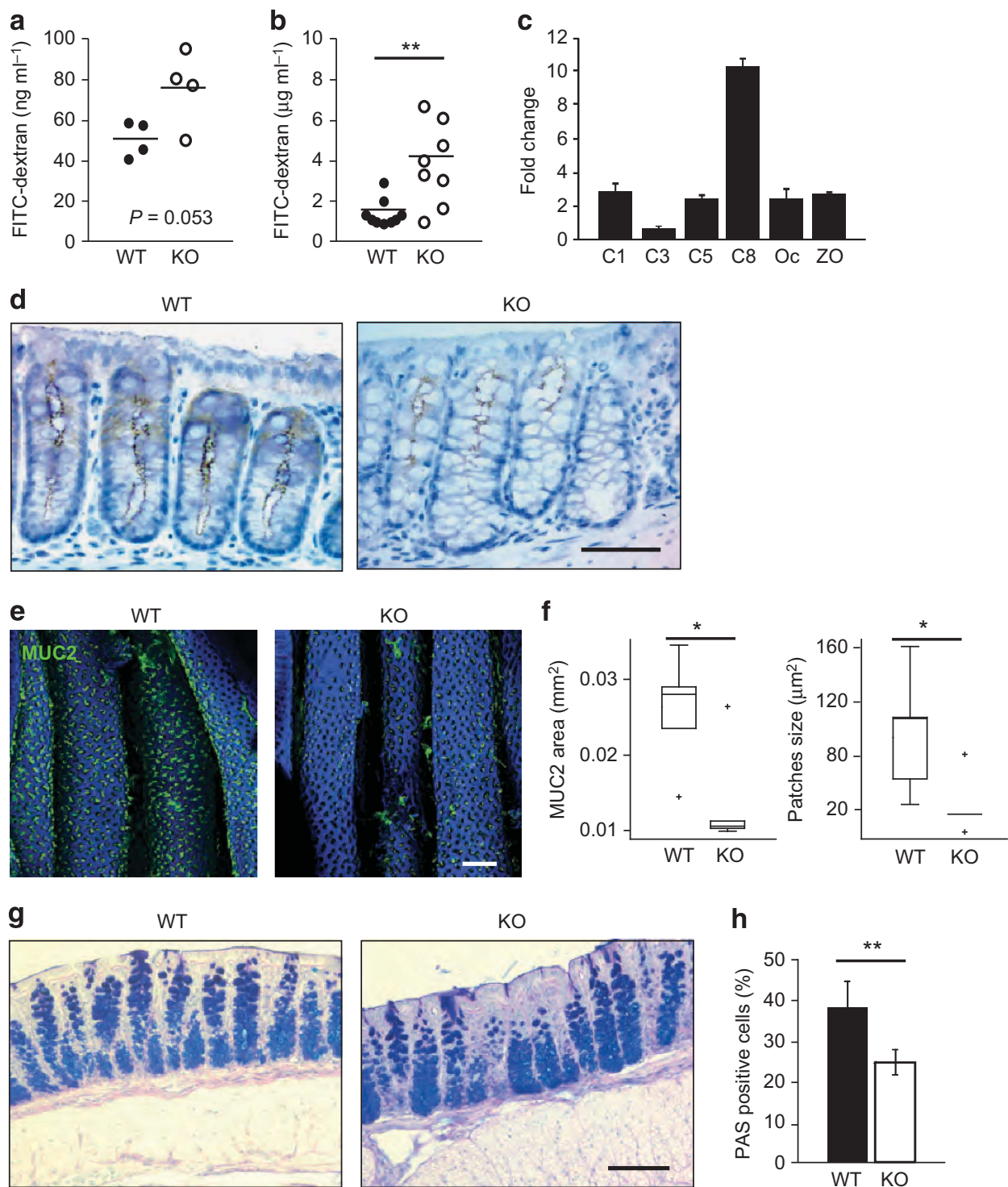

Figure 3 Compromised epithelial barrier function in $M m p-19^{-/-}$mice. (a, b) In vivo permeability of the mucosa of untreated (a) and DSS-treated (b) WT and $M m p-19^{-/-}$mice measured as FITC-dextran level in plasma $4 \mathrm{~h}$ after orogastric gavage. $n=4 ;{ }^{* \star} P<0.01$. (c) Comparison of mRNA expression for tight junction constituents (C1, claudin-1; C3, claudin-3; C5, claudin-5, C8; claudin-8; Oc, occludin; ZO, zonula occludens-1) in colonic mucosa of untreated WT vs. Mmp-19-1- mice. Expression was normalized to actin and expressed as fold change in WT samples. $n=3$. (d) Tissue sections of unchallenged WT and Mmp-19-1- deficient colonic mucosa stained with claudin-8 specific antibodies. Scale bar: $50 \mu \mathrm{m}$. (e) Confocal microscopy images of MUC2 expression in the colon mucus layer of WT and $\mathrm{Mmp}-19^{-/-}$mice. Scale bar: $100 \mu \mathrm{m}$. (f) Quantification of the area and size of MUC2positive patches. Box and whisker plots indicate the median (middle line in the box), 25th percentile (bottom line of the box), 75th percentile (top line of the box), and 2.5th and 97.5th percentiles (whiskers). (g,h) Comparison of presence of goblet cells in colon epithelia stained with Alcian blue and PAS. Scale bar: $50 \mu \mathrm{m},{ }^{\star \star} \mathrm{P}<0.01$.

(Supplementary Figure 2C). Moreover, infiltration of inflammatory cells, edema, and hyperregeneration indicated a well-established injury in Mmp-19 $19^{-/-}$mice (Supplementary Figure 2C).

Pro-inflammatory cytokine levels were substantially elevated in $M m p-19^{-1-}$ mice during the recovery phase, pointing to a more systemic immune response. In particular, IL-6 plasma concentrations were 10 -fold higher in $\mathrm{Mmp}-19^{-/-}$mice than in WT mice (Supplementary Figure 5). MCP-1, G-CSF, and KC were also significantly elevated in the plasma of $M m p-19^{-/-}$ mice. Highly increased levels of G-CSF indicate the initiation of a systemic response, potentially stimulating granulocyte production. Altogether, Mmp-19 $19^{-/-}$mice showed evidence of persistent inflammation.

\section{Impact of MMP-19-deficiency on migratory capacity of inflammatory cells}

CEC supernatants were injected into the dorsal air pouch of WT mice, and cellular infiltrate was analyzed after 6, 24 and $48 \mathrm{~h}$. The highest increase of cellularity was detected in CEC supernatants from $M m p-19^{-/-}$mice after $24 \mathrm{~h}$ (Figure 5a). The number of macrophages (Figure 5b) and neutrophils 

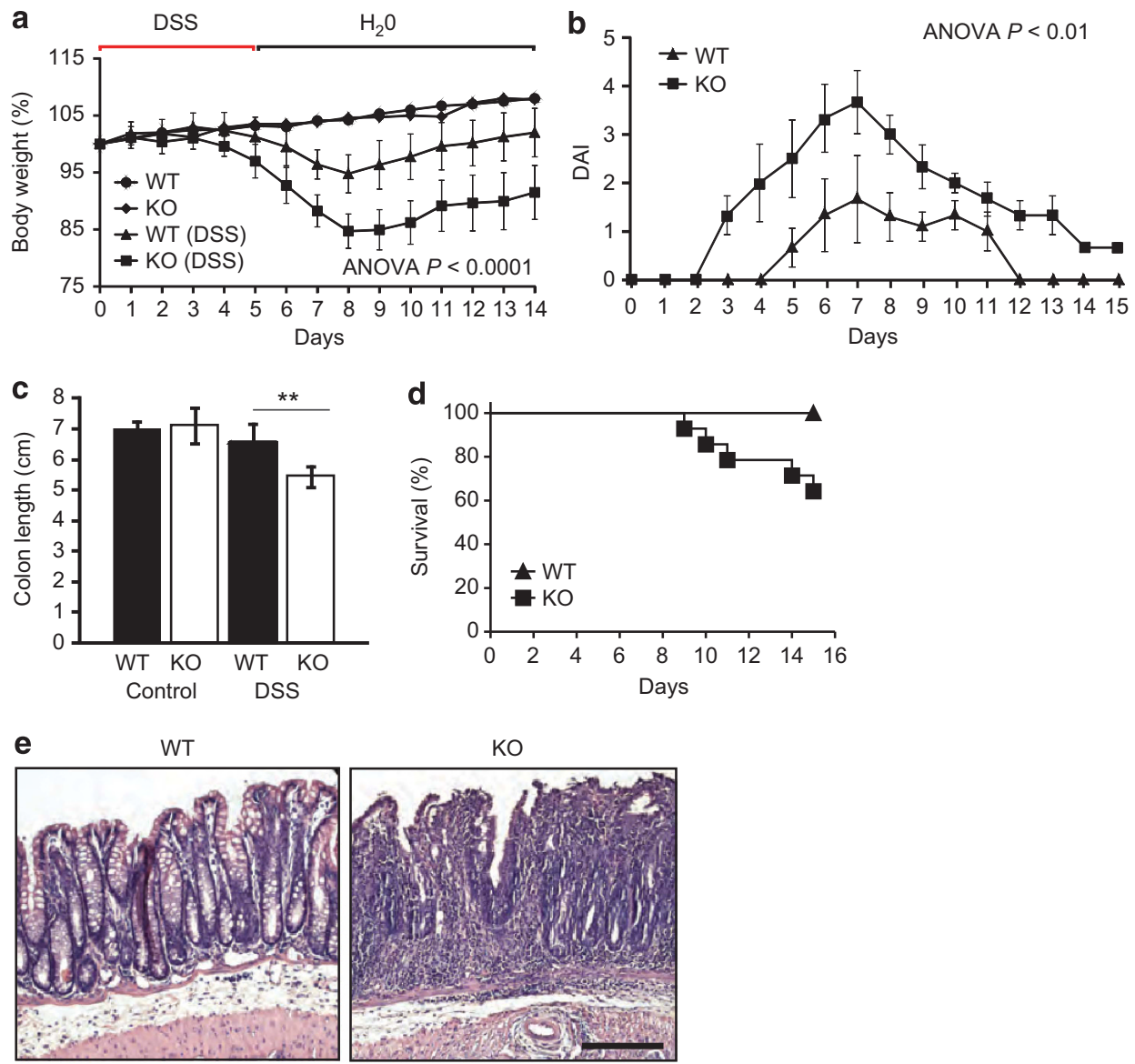

Figure 4 Impaired recovery from acute colitis in $\mathrm{Mmp}-19^{-/-}$mice. (a) Body weight changes during the course of acute colitis followed by a 10-day recovery phase as a percentage of original weight. $n=14$. (b) Disease severity in acute and healing phase of colitis as DAl. (c) Colon Length after recovery phase. $n=6$; ${ }^{* *} \mathrm{P}<0.01$. (d) Kaplan-Meier survival curves. (e) Representative H\&E-stained sections of distal colon obtained from DSS-treated mice after recovery (day 15); $M m p-19^{-1-}$ mice exhibit more severe inflammation and epithelial damage.

(Figure 5c) infiltrating the air pouch was significantly higher in the Mmp-19-1- CEC supernatants, which was consistent with the increased concentrations of chemoattractants (Supplementary Figure 5). Also, monocyte, granulocyte, $\mathrm{T}$ and B cell counts were higher in $M m p-19^{-/-}$mice (Supplementary Figure 6). Increased macrophage migration in response to chemoattractant-rich $M m p-19^{-1-}$ CEC supernatants was confirmed in the Transwell-chamber migration assay with the RAW264.7 macrophage cell line and the scratch wound healing assay with primary macrophages (Figure 5d).

The intrinsic impact of MMP-19 on the migratory capacity of inflammatory cells was assayed in the air-pouch model with zymosan challenge. FACS analysis of pouch exudates (Figure 5e) revealed comparable infiltration with granulocytes (LY6) and monocytes (CD14) but reduced numbers of Mmp-19-1- CD11b- and F4/80-positive macrophages. Peritoneal macrophages from $M m p-19^{-/-}$mice labeled with calcein AM also exhibited significantly decreased transmigration (Figure 5f). Altogether, these results suggest that the migration capacity of $M m p-19^{-/-}$macrophages is diminished; however, stronger pro-inflammatory response in Mmp-19 $19^{-/-}$mice is sufficient to override their compromised migratory potential.

\section{Wild-type bone marrow transplantation rescues phenotype of $\mathrm{Mmp}-19^{-1-}$ mice}

To address if Mmp-19 deficiency principally affects epithelial cells or blood-derived immune cells, reciprocal bone marrow (BM) transplantations were performed. The chimeric recipients were challenged with $2 \%$ DSS to induce acute colitis (Figure 6a and b). Strikingly, $M m p-19^{-/-}$mice transplanted with Venus bone marrow cells showed virtually no symptoms of acute colitis, in contrast to WT and $M m p-19^{-/-}$mice injected with Mmp-19-deficient cells. Mmp-19-1- recipients of WT cells displayed no weight loss, normal colon length (data not shown), significantly smaller and fewer ulcers (Figure $\mathbf{6 c}$ and data not shown), and no increase of macrophages (Figure 6d) and neutrophils (Figure 6e) in the mucosa. Levels of pro- and anti-inflammatory mediators in the plasma of rescued $M m p-19^{-/-}$mice were either comparable (KC, TNF $\alpha$ ) or significantly decreased (IL-4, IL-6) when compared to their non-rescued counterparts (Supplementary Figure 3B,C). These findings suggest that transplantation of WT bone marrow either ameliorated or nearly abrogated the exuberant inflammation in $M m p-19^{-/-}$mice. 

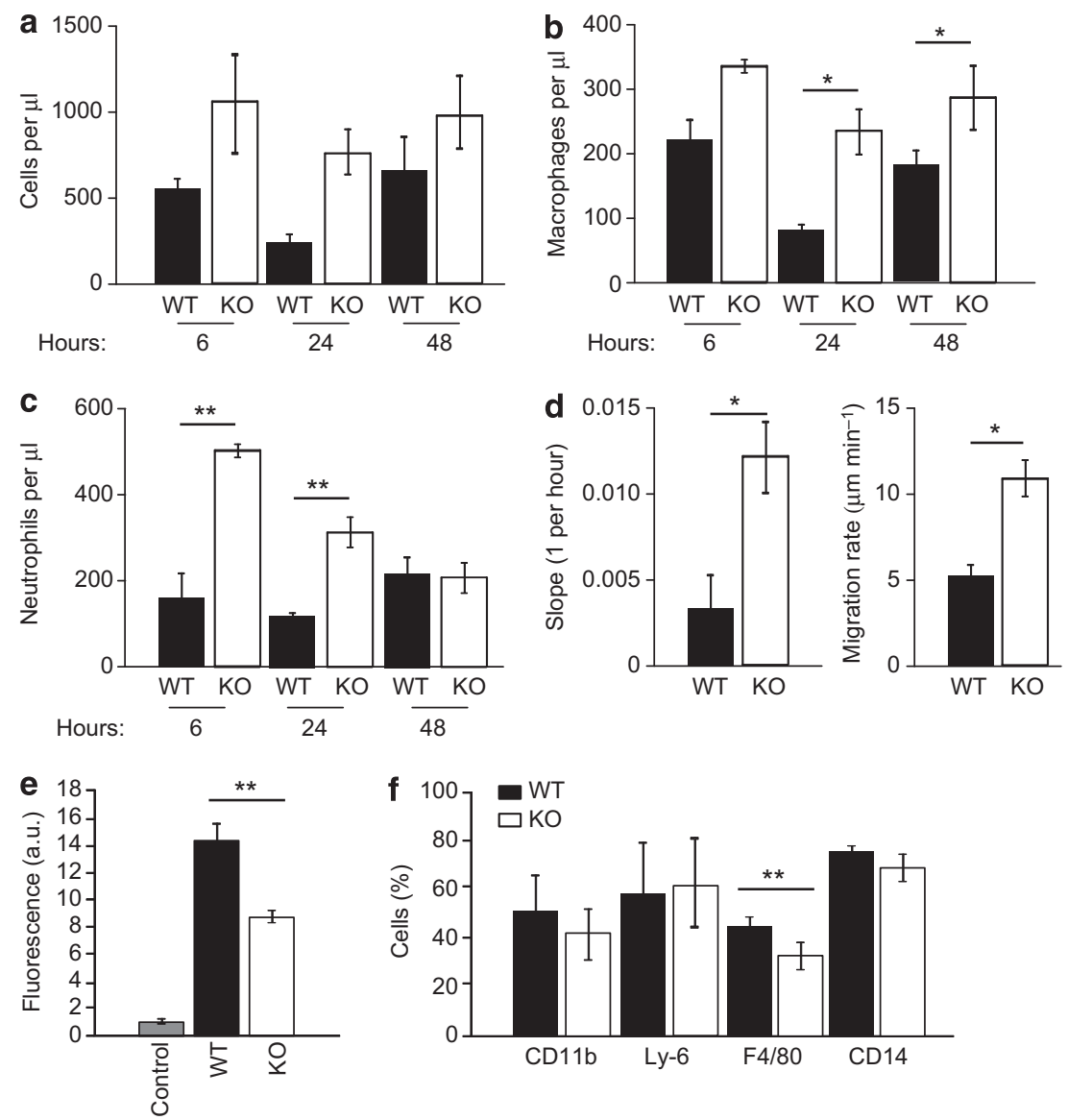

Figure 5 Enhanced migration of macrophages and neutrophils in the presence of media conditioned by Mmp-19-1- CEC in the air pouch model. (a-c)

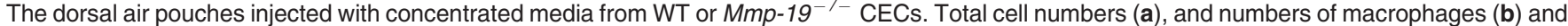
neutrophils (c) were determined using flow cytometry in exudates obtained 6,24 , and 48 h after injection. $n=3$; ${ }^{\star} P<0.05$, ${ }^{\star *} P<0.01$. (d) RAW 264.7 cells (left) were seeded on collagen IV coated Transwell inserts and their transmigration was observed for $6 \mathrm{~h}$ against WT or Mmp-19 ${ }^{-1-}$ CEC media. Primary macrophages (right) isolated from bone marrow were monitored for migration in a scratch wound closure assay in the presence of concentrated media from either WT or Mmp-19 ${ }^{-/-}$CEC. $n=3 ;{ }^{*} P<0.05$. (e) Migratory capacity of $M m p-19^{-1-}$ macrophages is markedly impeded. Calcein AM-labeled peritoneal macrophages of WT and $\mathrm{Mmp}-19^{-/-}$mice transmigrated through collagen IV coated membrane and fluorescence in the lower chamber was measured. (f) Air pouch model, migratory capacities of inflammatory cells. Inflammation was induced by zymosan. Pouch exudates were analyzed by FACS for the presence of granulocytes (Ly6G), macrophages (CD11b and F4/80) and monocytes (CD14).

\section{MMP-19 cleaves the chemokine domain of CX3CL1}

Mmp-19 deficiency had no effect on granulopoiesis and mobilization of neutrophils in response to G-CSF as demonstrated by daily administration of G-CSF (Figure 7a and $\mathbf{b}$ ) and thus we searched for potential mechanisms of deregulated neutrophil influx. We examined also CX3CL1 (fractalkine), a unique chemokine with adhesive and chemotactic properties that is expressed in epithelia. ${ }^{30-32}$ CX3CL1-CX3CR1 signaling is pivotal for the development of inflammatory conditions in intestinal walls ${ }^{33-35}$ and ablation of the receptor leads to a similar phenotype as observed in $\mathrm{Mmp}-19^{-/-}$mice. ${ }^{35}$ Colon tissue lysates and conditioned media from CECs were analyzed by immunoblotting using antibodies against the $\mathrm{N}$ - or C-terminus of CX3CL1. The N-terminal detection revealed a fragment of about $40 \mathrm{kDa}$ in $M m p-19^{-/-}$samples which was virtually absent in WT samples (Figure 7c; for full unedited gels see Supplementary Figure 7A). A soluble CX3CL1 variant of about $23 \mathrm{kDa}$ was considerably less abundant in supernatants from $M m p-19^{-/-}$mice (Figure 7c). Incubation of human
CX3CL1 with proteolytically active MMP-19 (GST-MMP-19 WT), but not with inactive mutant (GST-MMP-19 E213A), ${ }^{20}$ resulted in almost complete processing of the full-length CX3CL1 into three cleavage products of around 70, 20, and $15 \mathrm{kDa}$ (Figure 7d; for full unedited gels see Supplementary Figure 7B). MMP-19-dependent shedding of CX3CL1 was also confirmed in HeLa cells co-transfected with MMP-19 and CX3CL1 (Figure 7e and $\mathbf{f}$; for full unedited gels see Supplementary Figure 7C). Hence, we provided evidence that MMP-19 generates soluble chemokine domain of CX3CL1. Cell conditioned media (CCM) from HeLa cell lines were injected into the dorsal air pouch of WT mice, and cellular infiltrate was analyzed for neutrophils after 6 , and $24 \mathrm{~h}$ (Figure $7 \mathbf{g}$ ). Neutrophil numbers in CCM from cells overexpressing MMP-19 and CX3CL1 were significantly higher when compared to those obtained from CCM derived from cells transfected with CX3CL1 only. This mimics the delayed influx of neutrophils into lesions of $\mathrm{Mmp}-19^{-/-}$mice in acute DSS-induced colitis (Figure 2a) and might be linked to cleaved 
a

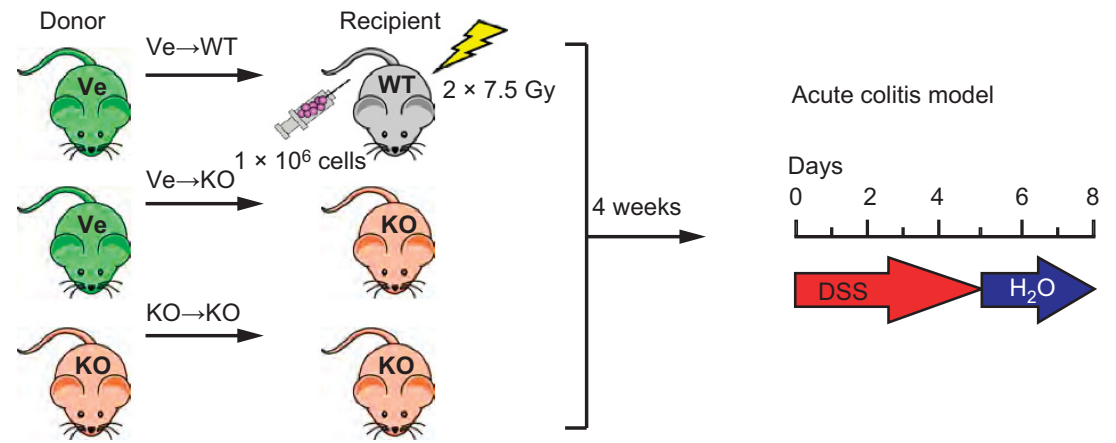

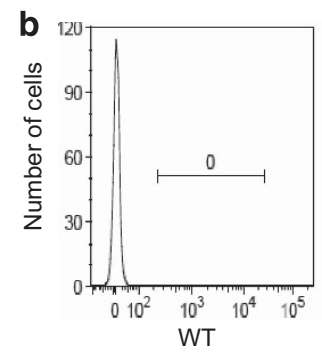

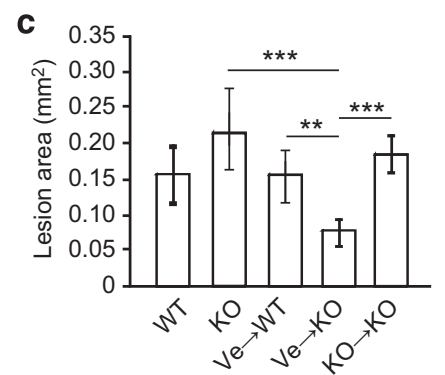

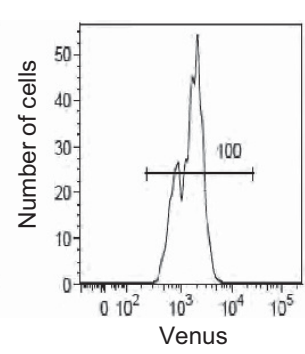

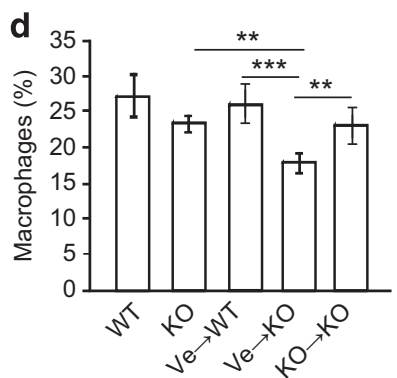

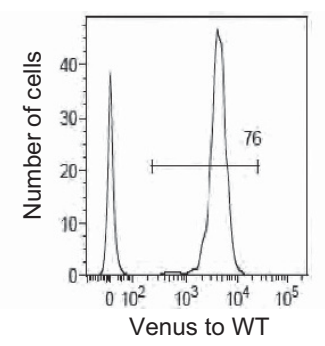
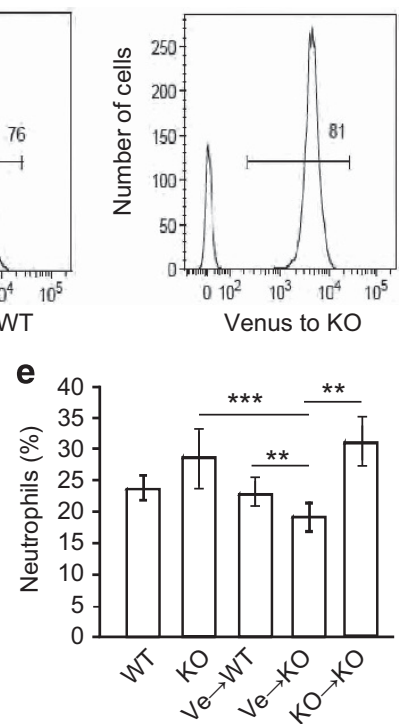

Figure 6 Bone marrow transplantation overcomes detrimental effect of $M m p-19$ deficiency in colitis. (a) Generation of bone marrow chimeric mice where donors and/or recipients were either Venus wild-type (Ve), WT or $\mathrm{Mmp}-19^{-/-}$mice. Chimeric recipients $(\mathrm{Ve} \rightarrow \mathrm{WT}, \mathrm{Ve} \rightarrow \mathrm{KO}$ and $\mathrm{KO} \rightarrow \mathrm{KO})$ were subjected to acute colitis induction. (b) Results of FACS analysis of transplantation efficiency displaying counts of original (Venus) or repopulating GFP positive cells. (c) Area of the ulcerative lesions in colon mucosa. $n=3-6$. (d) Counts of tissue macrophages stained for F4/80 expression and (e) neutrophils stained for MPO in 5 different fields per colon per mouse. The results represent three independent experiments, $n=3-5$, ${ }^{\star \star} P<0.01,{ }^{\star \star \star} P<0.001$.

chemokine domain of CX3CL1 in tissue (Figure 7c) and CCM (Figure $7 \mathbf{g}$ ), respectively. Of note, neutrophil numbers in CCM from cells over-expressing MMP-19 alone were comparable to those in CCM from cells transfected with MMP-19 and CX3CL1. This suggests that either MMP-19 sheds more of endogenous CX3CL1 from the walls of the subcutaneous air pouch or that cleaved CX3CL1 modulates neutrophil migration in conjunction with some other chemokines released by MMP- 19 .

\section{DISCUSSION}

In this study, we provide functional evidence that MMP-19 controls the inflammatory processes in the intestine and plays a protective role in colitis development. This phenotype partly resembles that of other MMPs. Mmp- $7^{-1-}$ mice showed delayed re-epithelialization and influx of neutrophils due to alteration of neutrophil chemokines. ${ }^{36}$ MMP-2 also exhibits some protective effect. However, MMP-9 had the opposite effect on the course of colitis, with the extent and severity of intestinal epithelial injury being significantly attenuated in the deficient mice. ${ }^{37,38} \mathrm{Mmp}-9^{-1-}$ mice exposed to DSS or $S$. typhimurium had dramatically reduced inflammation and mucosal injury and showed protection against acute colitis. ${ }^{38,39}$ However, bone marrow transplantation showed that loss of granulocyte-derived MMP-9 exacerbated colitis whereas general loss of MMP-9, including MMP-9 of epithelial origin, exhibited attenuation. ${ }^{39}$

MMP-19 appears to affect both the epithelium and the inflammatory response. The colonic epithelial barrier in Mmp-19 $19^{-1-}$ mice was extensively compromised during the recovery and chronic phases of colitis. Analysis of proteins involved in constituting tight junctions (TJs), and thus paracellular permeability homeostasis, revealed that the colonic epithelium of Mmp-19 $19^{-/}$mice expresses significantly less claudin-8. Claudin8 is expressed prevalently in the colon, ${ }^{40}$ and together with claudin-5 is responsible for sealing TJs, thus preventing back leakage in the most distal segments of tubular epithelia. ${ }^{41}$ The impact of MMP-19 on barrier function may also be due to processing of its substrates found in the basement membrane such as laminin $5 \gamma 2$ chain, nidogen- 1 , or tenascin $C^{20-22}$

To distinguish whether $M m p-19$ deficiency primarily impacts the epithelia or immune cells during colitis develop-

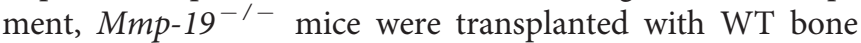



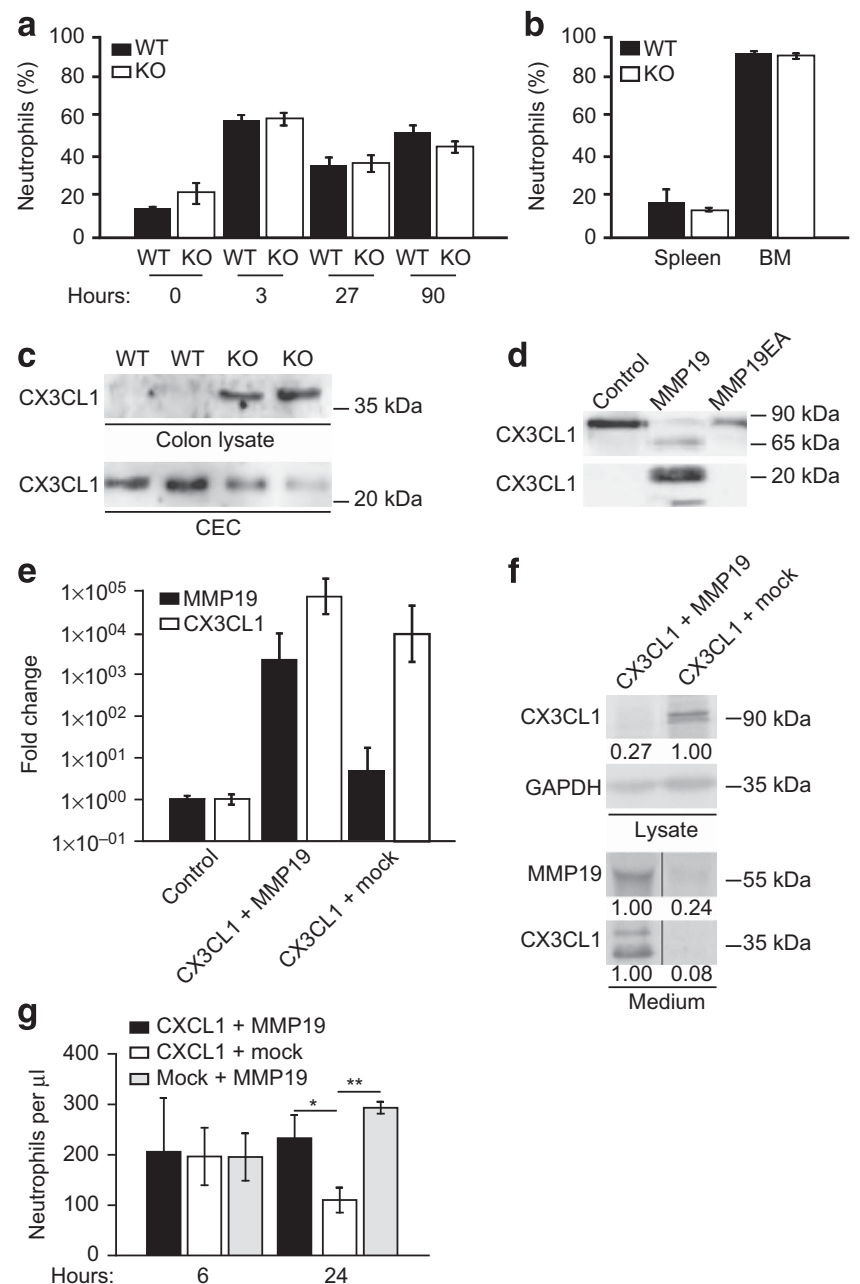

Figure 7 Processing of CX3CL1 by MMP-19 in vitro and in vivo. (a,b) WT Mice were injected daily with G-CSF for 4 days. Numbers of neutrophils (a) from peripheral blood were determined by FACS at 3,27 , and $90 \mathrm{~h}$ after the first injection. (b) Neutrophil numbers in bone marrow and spleen were determined on day 4. (c) Equal protein concentrations of colon lysate and equal volumes of concentrated CEC media were analyzed by immunoblotting with anti-CX3CL1 antibodies against $\mathrm{N}$-terminal epitope. (d) CX3CL1 is directly processed by MMP-19. Recombinant human CX3CL1 was incubated alone (control), in the presence of active (MMP19 ), or the inactive mutant (MMP-19EA) forms of MMP-19. CX3CL1 fragments of $\sim 70,20$ and $15 \mathrm{kDa}$ size exhibit different sizes to endogenous protein possibly due to post-translational modification (e.g. glycosylation; c and d). (e-g) HeLa cells were either untreated or transfected with CX3CL1, MMP-19, and empty EGFP (mock) expression constructs as indicated. Relative mRNA levels (e) and CX3CL1 shedding (f) were examined by qRT-PCR and immunoblotting, respectively. Numbers below lanes in (f) indicate relative band intensity (normalized to highest value). GAPDH, loading control. (g) Indicated HeLa cell conditioned media were injected into the dorsal air pouches of WT mice. Numbers of neutrophils were determined using flow cytometry in exudates obtained 6 , and $24 \mathrm{~h}$ after injection. $n=3$; ${ }^{*} P<0.05$, ${ }^{\star \star} P<0.01$.

marrow cells. These chimeric Mmp-19-1- mice developed only weak signs of colitis, resembling the disease progression of WT mice. This rescue effect could be explained by a neutrophil migration rate similar to that of WT mice and/or by significantly decreased plasma cytokine levels compared to the controls.
The dysregulated immune response in $M m p-19^{-/-}$mice and its bias towards inflammation is obvious during all phases of DSSinduced colitis. However, most striking is the slower kinetics of neutrophil influx in the acute phase, in which the number of neutrophils in $M m p-19^{-/-}$mice increased very slowly. As neutrophils do not express MMP-19, the defect in transmigration into the inflamed tissue was probably a consequence of a lack of expression of an appropriate chemoattractant in the epithelial cells or, mechanically, their removal due to excessive damage. Thus, MMP-19 seems to function as a mediator for neutrophil migration to wounded mucosa.

Besides neutrophils, MMP-19 exhibits a direct impact on migration of macrophages. Using the Transwell-chamber and air-pouch migration assays, we observed a lower migratory capacity of the Mmp-19 ${ }^{-1-}$ macrophages. This finding is in line with our previous data showing association of MMP-19 with the cell-surface of myeloid cells. ${ }^{18}$

The observed impaired primary immune reaction might originate from the inability of inflammatory cells to migrate towards the site of injury, either due to the unavailability of attracting mediators, or dysfunction of their signaling pathways. Several MMPs have been identified to be involved in such a control of inflammatory cells. MMP-8 was shown to cleave the N-terminus of both CXCL8/IL-8 and LIX to enhance chemotactic potency and $M m p-8^{-/-}$mice showed impaired recruitment of polymorphonuclear leukocytes in an LPS-induced inflammation model. ${ }^{42}$ Other studies have demonstrated roles for MMP-2 and MMP-9 in mobilization of CCL11/eotaxin, CCL7/MCP-3 and CCL17/ TARC. $^{43}$

As MMP-19 associates with the cell surface, we examined if MMP-19 is able to process CX3CL1, which is also expressed in the intestinal mucosa ${ }^{32,44}$ and serves as chemoattractant when the chemokine domain is shed by ADAMs. ${ }^{45,46}$ Although most studies showed that CX3CL1 exhibits its main chemoattractive activity towards monocytes, natural killer cells, and $\mathrm{T}$ cells, ${ }^{30,31}$ expression of its receptor CX3CR1 was also reported on neutrophils. ${ }^{47}$ Brand et al. ${ }^{33}$ reported not only the surface expression of CX3CR1 on neutrophils but also that CX3CL1 promotes accumulation of neutrophils in intestinal epithelia. The attraction of neutrophils might not only be a direct effect of CX3CL1 alone but it could further lead to upregulation of IL-8/ $\mathrm{KC}$ in intestinal epithelia; IL-8/KC are potent chemoattractants for neutrophils. We show for the first time that MMP-19 cleaves CX3CL1, and that CX3CL1 is not effectively released from colon explants of $M m p-19^{-/-}$mice. In comparison to ADAM17 that cleaves the CX3CL1 stalk proximal to the cell surface, MMP-19 cleaves off only the chemokine domain. The diminished MMP-19-dependent release of CX3CL1 could be partly responsible for dysregulated recruitment of neutrophils and other inflammatory cells. Reduced CX3CL1-mediated signaling might also lead to an impaired capacity of epithelia to recover as CX3CR1 in intestinal epithelial cells promotes cell proliferation. ${ }^{33}$ Interestingly, the altered CX3CL1-CX3CR1 axis appeared to be pivotal for mucosal immunity at the background of chronic colitis ${ }^{48}$ and CX3CR1 $1^{-/-}$mice exhibit 
impaired intestinal barrier and thinner mucus. ${ }^{49}$ Some aspects of our concept of the physiological role of MMP-19-dependent CX3CL1 cleavage remain speculative and need further investigation.

In conclusion, our data provide evidence that MMP-19 has a protective role in colitis by maintaining the colon epithelial barrier and controlling the recruitment of inflammatory cells. Moreover, we provided evidence that MMP-19 sheds the chemokine domain of CX3CL1 revealing thus another important activation component during intestinal inflammation and complexity of MMP19 role in colitis.

\section{METHODS}

Experimental animals. $M m p-19^{-/-}$mice were described previously. ${ }^{24}$ Venus mice were generated using transposon mediated mutagenesis by using Sleeping Beauty transposons (SB100) containing Venus under the CAGGS promoter (gift from L. Mates, MDC Berlin, Germany). The majority of tissues and cell types in Venus mice expressed GFP; the animals were otherwise phenotypically indistinguishable from WT mice. Genotyping is described in Supplementary Material.

DSS-induced colitis model and disease activity scoring. Only males were used for all experiments as a significant gender bias in susceptibility to DSS-induced colitis was reported. ${ }^{36}$ Acute colitis was induced by administration of $2 \%$ dextran sulphate sodium (DSS; TdB Consultancy) in the drinking water for 6 days, after which mice received 2 days resting period before they were killed. Mice drinking normal water were used as control. Body weight, disease activity index (DAI: calculated using the loss of body weight, intensity of bleeding (Hemoccult Fecal Occult Blood Test, Beckman Coulter) and stool consistency. The recovery phase of colitis was induced by DSS administration for 5 days followed by a 10 days recovery period. In the model of chronic colitis, the latter procedure was repeated twice. DAI scoring is described in Supplementary Material.

Murine colon organ culture. Whole colons were excised and flushed with PBS containing penicillin and streptomycin (PAA). Biopsies from the distal colon $(3 \times 8 \mathrm{~mm})$ were excised and the colon explant culture (CEC) set up for $24 \mathrm{~h}$. Culture supernatants were assessed for cytokines/chemokines by ELISA or Bio-Plex Suspension Array System (Bio-Rad Laboratories).

Bone marrow transplantation. Transplants were performed as previously described. ${ }^{27}$ Briefly, four-week-old recipients were irradiated with $7.5 \mathrm{~Gy}$ at two doses with $5 \mathrm{~h}$ interval. The same day, $1-2 \times 10^{6}$ unsorted BM cells isolated from femur of the donors, were injected via the tail vein. Four weeks after the transplantation, acute colitis was induced as described above.

Gelatin zymography. In brief, gels were incubated for $24 \mathrm{~h}$ at $37^{\circ} \mathrm{C}$ and stained with $0.5 \%$ Coomassie Blue R-250 (Sigma-Aldrich). Regions representing the gelatinase activity of MMP-2 and MMP-9 were quantified using AIDA Image Analyser Software (Raytest).

Myeloperoxidase activity measurement. Myeloperoxidase (MPO) activity was measured in snap-frozen samples from distal colon. Equal aliquots of tissue samples were homogenized in MPO buffer $\left(50 \mathrm{mM}\right.$ potassium phosphate buffer $\left(\mathrm{KPO}_{4}\right)$, $\mathrm{pH} 6.0$, containing $0.5 \%$ hexadecyltrimethylammonium bromide $\left(\mathrm{C}_{19} \mathrm{H}_{42} \mathrm{BrN}\right)$, incubated at $60^{\circ} \mathrm{C}$ for $2 \mathrm{~h}$. Diluted, clarified samples were mixed with the $\mathrm{O}$-dianisidine and MPO activity was assayed as described elsewhere.

In vivo permeability assay. To measure intestinal permeability, FITC-dextran 4 (4000 MW; TdB Consultancy) dissolved in PBS was administered by oral gavage $(0.6 \mathrm{~g} / \mathrm{kg}$ body weight $)$ to mice fasted for $4 \mathrm{~h}$ prior to the experiment. Blood was obtained by retro-orbital bleeding into heparin coated tubes (Microvette CB 300, Sarstedt).

Expression of MUC2 in colon mucus layer. Distal parts of colon tubes were fixed with methanol based Carnoy's fixative and opened tissues were stained with anti MUC2 antibody followed by Alexa 488 conjugated secondary antibody. Subsequently, specimens were soaked with glycerol and screened with Leica SP5 confocal microscope. Total area, number and average size of MUC2 positive patches were used for statistical comparison.

CX3CL1 processing in vitro. The cleavage of CX3CL1 with human GST-MMP-19 and inactive MMP-19 mutant (MMP-19EA) was assayed as described. ${ }^{19}$ In brief, $1 \mu \mathrm{g}$ of human CX3CL1 (R\&D Systems) was incubated with recombinant MMP-19 in TNC buffer (50 mM Tris- $\mathrm{HCl}, 150 \mathrm{mM} \mathrm{NaCl}, 5 \mathrm{mM} \mathrm{MgCl}_{2}, 5 \mathrm{mM} \mathrm{CaCl}_{2}, \mathrm{pH}$ 7.4) containing $10 \mu \mathrm{M} \mathrm{ZnCl} 2$ with $4 \mu \mathrm{g}$ GST-MMP-19 at $37^{\circ} \mathrm{C}$ for $35 \mathrm{~h}$.

Dorsal air pouch. Assay was performed as described. ${ }^{50}$ At day $6,1 \mathrm{ml}$ of WT, Mmp-19-1- CEC supernatant (diluted in PBS) or PBS alone (control) was injected into the pouch. Mice were killed 6, 24 or $48 \mathrm{~h}$ after injection and infiltrated white blood cells were analyzed by flow cytometry.

Statistics. All parametric data are presented as mean \pm s.d. Statistical analyses were performed with GraphPad Prism (GraphPad Software). Differences between 2 groups were tested using a two-tailed unpaired Student's $t$ test. Two-tailed one-way ANOVA or Kruskal-Wallis test with Dunn's post-test was used for comparison of multiple groups when appropriate. Significance was determined at the level of $P<0.05 .{ }^{\star} P<0.05$, ${ }^{*} P<0.01,{ }^{* *} P<0.001$; the $\mathrm{n}$ value is specified in the figure legends.

Study approval. All animal studies were ethically reviewed and performed in accordance with European directive 86/609/EEC and were approved by the Czech Central Commission for Animal Welfare.

Full-length Material and Methods are described in Supplementary Material.

SUPPLEMENTARY MATERIAL is linked to the online version of the paper at http://www.nature.com/mi

\section{ACKNOWLEDGMENTS}

We thank Lenka Sarnova for her outstanding technical assistance and to Trevor Epp for critical reading of the manuscript. Financial support was given to RS by GACR (P302/11/2048 and P303-10-2044) Academy of Sciences of the Czech Republic (RVO 68378050), and the project BIOCEV-Biotechnology and Biomedicine Centre of the Academy of Sciences and Charles University' (CZ.1.05/1.1.00/02.0109) from the European Regional Development Fund, and GAUK (project 64212), and to $\mathrm{MH}$ (CZ.1.05/2.1.00/01.0030). Publicly accessible material: MMP-19 deficient mouse strain B6.MMP19 (B6.129P2-Mmp19tm1Rase/Ph) is deposited in INFRAFRONTIER/EMMA repository under EM:06786.

\section{AUTHOR CONTRIBUTION}

Study concept and design: R.S., R.B. Acquisition of data: R.B., J.T., M.G., I.K., M.D., J.S., I.M.B., O.Z., P.K., K.C., T.K., J.P. Analysis and interpretation of data: R.B., J.T., M.G., I.K., M.D., J.S., I.M.B., O.Z., P.K., K.C., T.K, J.P., R.S. Drafting of the manuscript: R.B., J.T., M.G., R.S. Critical revision of the manuscript for important intellectual content: all authors. Statistical analysis: R.B., J.T., M.G., I.K., M.D., J.S., I.M.B., O.Z., P.K., K.C., T.K., F.S. Obtained funding: R.S. Technical or material support: V.K., K.-H.H., M.H. Study supervision: R.S., M.G.

\section{DISCLOSURE}

The authors declare no conflict of interest.

c 2016 Society for Mucosal Immunology 


\section{REFERENCES}

1. Podolsky, D.K. Inflammatory bowel disease. N. Engl. J. Med. 347, 417-429 (2002).

2. Xavier, R. J.\& Podolsky, D.K. Unravelling the pathogenesis of inflammatory bowel disease. Nature 448, 427-434 (2007).

3. Gao, Q. et al. Expression of matrix metalloproteinases-2 and - 9 in intestinal tissue of patients with inflammatory bowel diseases. Dig. Liver Dis. 37, 584-592 (2005).

4. Kirkegaard, T., Hansen, A., Bruun, E. \& Brynskov, J. Expression and localisation of matrix metalloproteinases and their natural inhibitors in fistulae of patients with Crohn's disease. Gut 53, 701-709 (2004).

5. Medina, C. \& Radomski, M. W. Role of matrix metalloproteinases in intestinal inflammation. J. Pharmacol. Exp. Ther. 318, 933-938 (2006).

6. Murphy, S. F., Kwon, J.H. \& Boone, D.L. Novel players in inflammatory bowel disease pathogenesis. Curr. Gastroenterol. Rep. 14, 146-152 (2012).

7. Naito, Y. et al. An orally active matrix metalloproteinase inhibitor, ONO4817, reduces dextran sulfate sodium-induced colitis in mice. Inflamm Res. 53, 462-468 (2004).

8. Naito, Y. \& Yoshikawa, T. Role of matrix metalloproteinases in inflammatory bowel disease. Mol. Aspects Med. 26, 379-390 (2005).

9. Pender, S. L. \& MacDonald, T. T. Matrix metalloproteinases and the gut-new roles for old enzymes. Curr. Opin. Pharmacol. 4, 546-550 (2004).

10. von Lampe, B., Barthel, B., Coupland, S.E., Riecken, E.O. \& Rosewicz, S. Differential expression of matrix metalloproteinases and their tissue inhibitors in colon mucosa of patients with inflammatory bowel disease. Gut 47, 63-73 (2000).

11. Pender, S.L., Tickle, S.P., Docherty, A.J., Howie, D., Wathen, N.C. \& MacDonald, T.T. A major role for matrix metalloproteinases in Tcell injury in the gut. J. Immunol. 158, 1582-1590 (1997).

12. Huang, T.Y. et al. Minocycline attenuates experimental colitis in mice by blocking expression of inducible nitric oxide synthase and matrix metalloproteinases. Toxicol. Appl. Pharmacol. 237, 69-82 (2009).

13. Pendas, A.M. et al. Identification and characterization of a novel human matrix metalloproteinase with unique structural characteristics, chromosomal location, and tissue distribution. J. Biol. Chem. 272, 42814286 (1997).

14. Cossins, J., Dudgeon, T.J., Catlin, G., Gearing, A.J. \& Clements, J.M. Identification of MMP-18, a putative novel human matrix metalloproteinase. Biochem. Biophys. Res. Commun. 228, 494-498 (1996).

15. Bister, V. et al. Matrilysins-1 and -2 (MMP-7 and -26) and metalloelastase (MMP-12), unlike MMP-19, are up-regulated in necrotizing enterocolitis. J. Pediatr. Gastroenterol. Nutr. 40, 60-66 (2005).

16. Cervinkova, M. et al. Differential expression and processing of matrix metalloproteinase 19 marks progression of gastrointestinal diseases. Folia Biol. (Praha) 60, 113-122 (2014).

17. Sedlacek, R. et al. Matrix metalloproteinase MMP-19 (RASI-1) is expressed on the surface of activated peripheral blood mononuclear cells and is detected as an autoantigen in rheumatoid arthritis. Immunobiology $\mathbf{1 9 8 ,}$ 408-423 (1998).

18. Mauch, S., Kolb, C., Kolb, B., Sadowski, T. \& Sedlacek, R. Matrix metalloproteinase-19 is expressed in myeloid cells in an adhesion-dependent manner and associates with the cell surface. J. Immunol. 168, 1244-1251 (2002).

19. Sadowski, T., Dietrich, S., Koschinsky, F. \& Sedlacek, R. Matrix metalloproteinase 19 regulates insulin-like growth factor-mediated proliferation, migration, and adhesion in human keratinocytes through proteolysis of insulin-like growth factor binding protein-3. Mol. Biol. Cell 14, 4569-4580 (2003).

20. Sadowski, T. et al. Matrix metalloproteinase 19 processes the laminin 5 gamma 2 chain and induces epithelial cell migration. Cell. Mol. Life Sci. 62, 870-880 (2005).

21. Titz, B., Dietrich, S., Sadowski, T., Beck, C., Petersen, A. \& Sedlacek, R. Activity of MMP-19 inhibits capillary-like formation due to processing of nidogen-1. Cell. Mol. Life Sci. 61, 1826-1833 (2004).

22. Stracke, J.O. et al. Matrix metalloproteinases 19 and 20 cleave aggrecan and cartilage oligomeric matrix protein (COMP). FEBS Lett. 478, 52-56 (2000).
23. Stracke, J.O. et al. Biochemical characterization of the catalytic domain of human matrix metalloproteinase 19. Evidence for a role as a potent basement membrane degrading enzyme. J. Biol. Chem. 275, 14809-14816 (2000).

24. Beck, I.M. et al. MMP19 is essential for T cell development and $\mathrm{T}$ cell-mediated cutaneous immune responses. PLoS One 3, e2343 (2008).

25. Gueders, M.M. et al. Matrix metalloproteinase-19 deficiency promotes tenascin-C accumulation and allergen-induced airway inflammation. Am. J. Respir. Cell Mol. Biol. 43, 286-295 (2010).

26. Jirouskova, M. et al. Hepatoprotective effect of MMP-19 deficiency in a mouse model of chronic liver fibrosis. PLoS One 7, e46271 (2012).

27. $\mathrm{Yu}, \mathrm{G}$. et al. Matrix metalloproteinase-19 is a key regulator of lung fibrosis in mice and humans. Am. J. Respir. Crit. Care Med. 186, 752-762 (2012).

28. Jost, M. et al. Earlier onset of tumoral angiogenesis in matrix metalloproteinase-19-deficient mice. Cancer Res. 66, 5234-5241 (2006).

29. Chan, K.C. et al. Catalytic activity of Matrix metalloproteinase-19 is essential for tumor suppressor and anti-angiogenic activities in nasopharyngeal carcinoma. Int. J. Cancer 129, 1826-1837 (2011).

30. Bazan, J.F. et al. A new class of membrane-bound chemokine with a CX3C motif. Nature 385, 640-644 (1997).

31. Jones, B.A., Beamer, M. \& Ahmed, S. Fractalkine/CX3CL1: a potential new target for inflammatory diseases. Mol. Interv. 10, 263-270 (2010).

32. Muehlhoefer, A. et al. Fractalkine is an epithelial and endothelial cell-derived chemoattractant for intraepithelial lymphocytes in the small intestinal mucosa. J. Immunol. 164, 3368-3376 (2000).

33. Brand, S., Sakaguchi, T., Gu, X., Colgan, S.P. \& Reinecker, H.C. Fractalkine-mediated signals regulate cell-survival and immune-modulatory responses in intestinal epithelial cells. Gastroenterology 122, 166-177 (2002).

34. Kostadinova, F.I., Baba, T., Ishida, Y., Kondo, T., Popivanova, B.K. \& Mukaida, N. Crucial involvement of the CX3CR1-CX3CL1 axis in dextran sulfate sodium-mediated acute colitis in mice. J. Leukoc. Biol. 88, 133-143 (2010).

35. Medina-Contreras, O. et al. CX3CR1 regulates intestinal macrophage homeostasis, bacterial translocation, and colitogenic Th17 responses in mice. J. Clin. Invest. 121, 4787-4795 (2011).

36. Swee, M., Wilson, C.L., Wang, Y., McGuire, J.K. \& Parks, W.C. Matrix metalloproteinase-7 (matrilysin) controls neutrophil egress by generating chemokine gradients. J. Leukoc. Biol. 83, 1404-1412 (2008).

37. Garg, P. et al. Selective ablation of matrix metalloproteinase-2 exacerbates experimental colitis: contrasting role of gelatinases in the pathogenesis of colitis. J. Immunol. 177, 4103-4112 (2006).

38. Santana, A. et al. Attenuation of dextran sodium sulphate induced colitis in matrix metalloproteinase-9 deficient mice. World J. Gastroenterol. 12, 6464-6472 (2006).

39. Castaneda, F.E. et al. Targeted deletion of metalloproteinase 9 attenuates experimental colitis in mice: central role of epithelial-derived MMP. Gastroenterology 129, 1991-2008 (2005).

40. Fujita, $\mathrm{H}$. et al. Differential expression and subcellular localization of claudin-7, -8, -12, -13, and -15 along the mouse intestine. J. Histochem. Cytochem. 54, 933-944 (2006).

41. Amasheh, S. et al. Tight junction proteins as channel formers and barrier builders. Ann. N Y Acad. Sci. 1165, 211-219 (2009).

42. Tester, A.M. et al. LPS responsiveness and neutrophil chemotaxis in vivo require PMN MMP-8 activity. PLoS One 2, e312 (2007).

43. Corry, D.B. et al. Overlapping and independent contributions of MMP2 and MMP9 to lung allergic inflammatory cell egression through decreased CC chemokines. FASEB J. 18, 995-997 (2004).

44. Lucas, A.D. et al. The transmembrane form of the CX3CL1 chemokine fractalkine is expressed predominantly by epithelial cells in vivo. Am. J. Pathol. 158, 855-866 (2001).

45. Garton, K.J. et al. Tumor necrosis factor-alpha-converting enzyme (ADAM17) mediates the cleavage and shedding of fractalkine (CX3CL1). J. Biol. Chem. 276, 37993-38001 (2001).

46. Hundhausen, C. et al. The disintegrin-like metalloproteinase ADAM10 is involved in constitutive cleavage of $\mathrm{CX} 3 \mathrm{CL} 1$ (fractalkine) and regulates CX3CL1-mediated cell-cell adhesion. Blood 102, 1186-1195 (2003). 
47. Combadiere, C., Salzwedel, K., Smith, E.D., Tiffany, H.L., Berger, E.A. \& Murphy, P.M. Identification of CX3CR1. A chemotactic receptor for the human CX3C chemokine fractalkine and a fusion coreceptor for HIV-1. J. Biol. Chem. 273, 23799-23804 (1998).

48. Kayama, H. et al. Intestinal CX3C chemokine receptor 1(high) (CX3CR1(high)) myeloid cells prevent T-cell-dependent colitis. Proc. Natl Acad. Sci. USA 109, 5010-5015 (2012).
49. Schneider, K.M. et al. CX3CR1 is a gatekeeper for intestinal barrier integrity in mice: Limiting steatohepatitis by maintaining intestinal homeostasis. Hepatology 62, 1405-1416 (2015).

50. Sin, Y.M., Sedgwick, A.D., Chea, E.P. \& Willoughby, D.A. Mast cells in newly formed lining tissue during acute inflammation: a six day air pouch model in the mouse. Ann. Rheum. Dis. 45, 873-877 (1986). 